\title{
Suppression of multipacting in high power RF couplers operating with superconducting cavities

\author{
P.N. Ostroumov ${ }^{1}$, S. Kazakov ${ }^{2}$, D. Morris ${ }^{1}$, T. Larter ${ }^{1}$, A.S. Plastun ${ }^{1}$, J. Popielarski ${ }^{1}$, J. Wei ${ }^{1}$,
} T. $\mathrm{Xu}^{1}$

${ }^{1}$ Facility for Rare Isotope Beams (FRIB), Michigan State University, East Lansing, MI 48824, USA

\author{
${ }^{2}$ Fermi National Accelerator Laboratory, Batavia, IL 60510, USA
}

\begin{abstract}
Capacitive input couplers based on a $50 \Omega$ coaxial transmission line are frequently used to transmit RF power to superconducting (SC) resonators operating in CW mode. It is well known that coaxial transmission lines are prone to multipacting phenomenon in a wide range of RF power level and operating frequency. The Facility for Rare Isotope Beams (FRIB) being constructed at Michigan State University includes two types of quarter wave SC resonators (QWR) operating at $80.5 \mathrm{MHz}$ and two types of half wave SC resonators (HWR) operating at 322 MHz. As was reported in ref. [1] a capacitive input coupler used with HWRs was experiencing strong multipacting that resulted in a long conditioning time prior the cavity testing at design levels of accelerating fields. We have developed an insert into $50 \Omega$ coaxial transmission line that provides opportunity to bias the RF coupler antenna and protect the amplifier from the bias potential in the case of breakdown in DC isolation. Two of such devices have been built and are currently used for the off-line testing of 8 HWRs installed in the cryomodule.
\end{abstract}

\section{Introduction}

The first cryomodule comprising eight superconducting $\beta_{\mathrm{OPT}}=0.53$ HWRs for the FRIB Linac has been assembled recently and it is undergoing off-line cold and RF testing. The SC cavities have been equipped with original RF couplers [1] shown in Fig. 1. The couplers are designed to transmit $5 \mathrm{~kW} \mathrm{CW}$ power to the resonator operating under the beam loading. These couplers have shown strong electron loading due to multipacting during the vertical testing of SC cavities. At FRIB, the procedure of coupler processing includes off-line room temperature conditioning in the travelling wave regime in a specially developed conditioning system [2]. The conditioning of these couplers takes on average about 60 hours until the $20 \mathrm{~kW}$ RF power is successfully transmitted with $20 \%$ duty cycle. During the conditioning, significant heating of the coupler outer conductor has been observed.

\section{Development of DC bias insert}

The cryomodule is installed inside the concrete bunker to provide effective shielding from Xrays generated during the conditioning and operation of SC cavities. Two $8 \mathrm{~kW} \mathrm{RF}$ amplifiers are located on the top of the bunker. 3-1/8" $50 \Omega$ rigid coaxial lines are used to transmit RF 


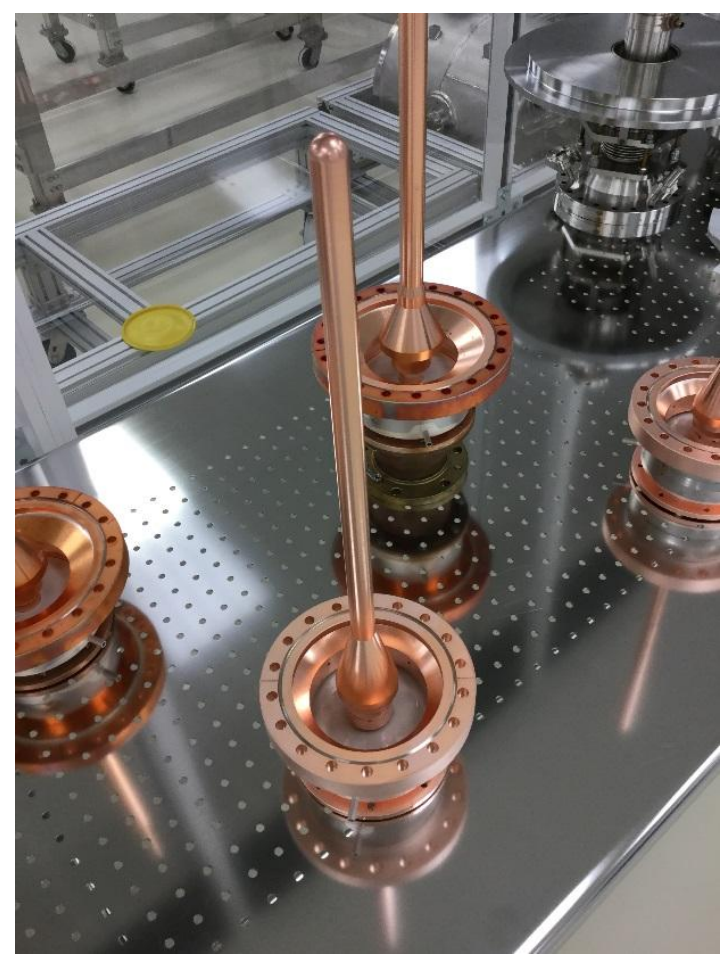

Fig. 1. The original FRIB power coupler antenna with the ceramic window (on the front) and outer conductor with the interface flange (on the back).

power from the amplifier to the HWRs. After cooling down to $4 \mathrm{~K}$, superconducting resonators and RF couplers require prolonged RF conditioning. The eventual goal of the resonator conditioning is to achieve phase-locked stable operation at accelerating field of $8.14 \mathrm{MV} / \mathrm{m}$, which is $10 \%$ higher than the design accelerating field for $\beta_{\mathrm{OPT}}=0.53 \mathrm{HWRs}$. To provide such an operation, the RF coupler and resonator itself must be multipactor-free in the wide range of frequency (or phase) difference between the generator and resonator. As soon as we have started resonator testing, we noticed significant pressure increase in the coupler which accompanied with temperature increase of the coupler outer conductor. Obviously, the multipacting has returned despite of conditioning of couplers in traveling wave regime at room temperature. The design accelerating field has been achieved with $1.9 \mathrm{~kW}$ forward $\mathrm{RF}$ power at the cavity resonance (see Table 1) after several days of RF coupler conditioning. However, the operation within the resonator bandwidth has resulted in strong multipacting preventing phase locked operation. Therefore, we have decided to develop and install a device which allows us to apply bias voltage to the RF coupler antenna.

Table 1. RF power required for testing and operation of HWRs.

\begin{tabular}{|l|l|l|}
\hline & Parameter & Value \\
\hline 1 & External quality factor, Q & $9.7 \times 10^{6}$ \\
\hline 2 & RF power to reach design accelerating field without beam & $1.9 \mathrm{~kW}$ \\
\hline 3 & RF power to reach 10\% higher than the design accelerating field with beam & $5.0 \mathrm{~kW}$ \\
\hline 4 & Available RF power & $8.0 \mathrm{~kW}$ \\
\hline
\end{tabular}


The full suppression of multipacting process in the capacitive RF couplers can be achieved by applying DC voltage into the antenna through the inner conductor of the coaxial line [3-5]. We have developed a DC bias insert (DC bias Tee) into the standard 3-1/8" coaxial transmission line as shown in Fig. 2. The principal of operation is as follows. The DC blocking capacitor is created by isolated overlaying inner conductors as shown in Fig. 3. The isolation is provided with three layers of 50- $\mu \mathrm{m}$-thick Kapton tape. The DC insert includes a quarter wave transformer (QWT) assembled from 3-1/8" coaxial transmission line sections. The QWT, a $\lambda / 4$-short, serves for two purposes: (1) to apply the bias potential into the inner conductor section connected to the RF coupler antenna as shown in Fig. 2 and (2) to prevent the application of the bias potential on RF amplifier output circuits in the case of short-circuit in the capacitor due to breakdown. The DC blocking capacitance can be calculated as:

$$
C=\frac{2 \pi \varepsilon \varepsilon_{0} l}{\ln \left(\frac{b}{a}\right)},
$$

where $\varepsilon$ is the relative dielectric permittivity of the film, $l$ is the capacitor length, $b$ and $a$ are the outer and inner diameter, correspondingly, of the inner conductor forming a capacitance as shown in Fig. 3. In our application, the gap in the capacitor was $\sim 0.15 \mathrm{~mm}$ with 3 layers of 50$\mu$ m-thick Kapton film which results in $C=196 \mathrm{pF}$. Due to the accuracy of fabrication of the transmission line parts, this capacitance can differ from the calculated value.

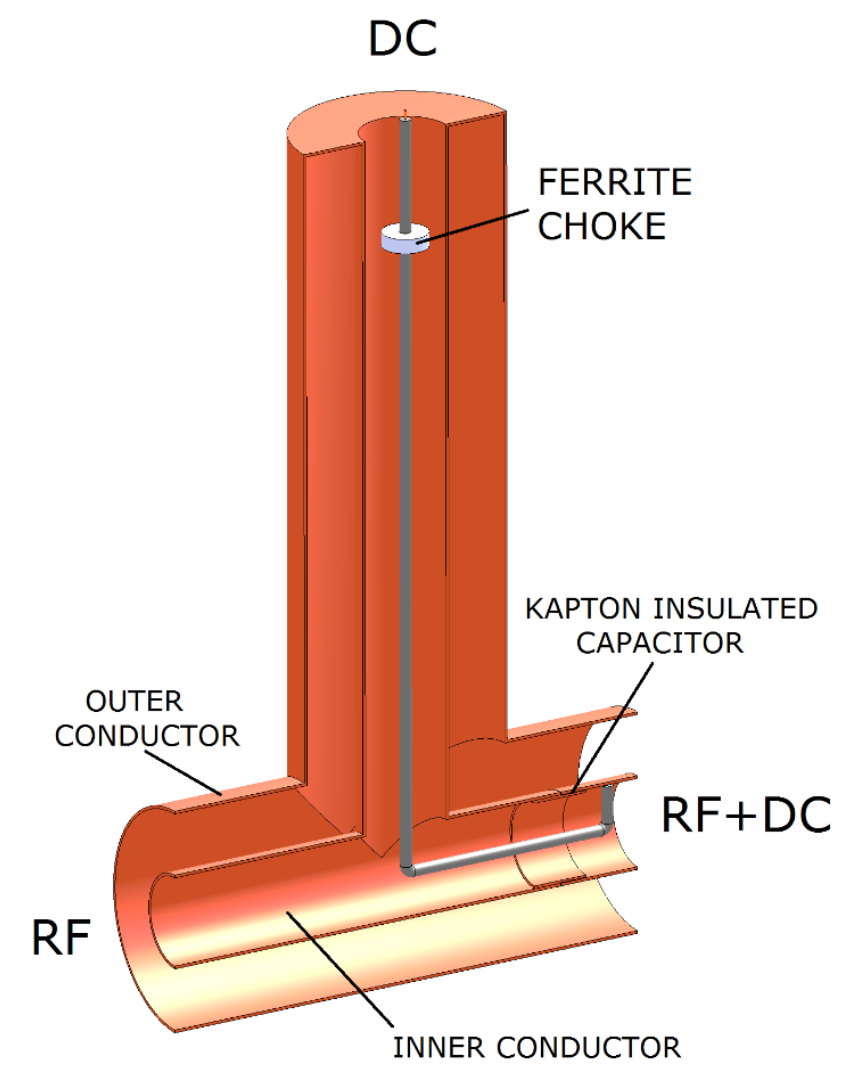

Figure 2: Schematic view of the bias tee model. 


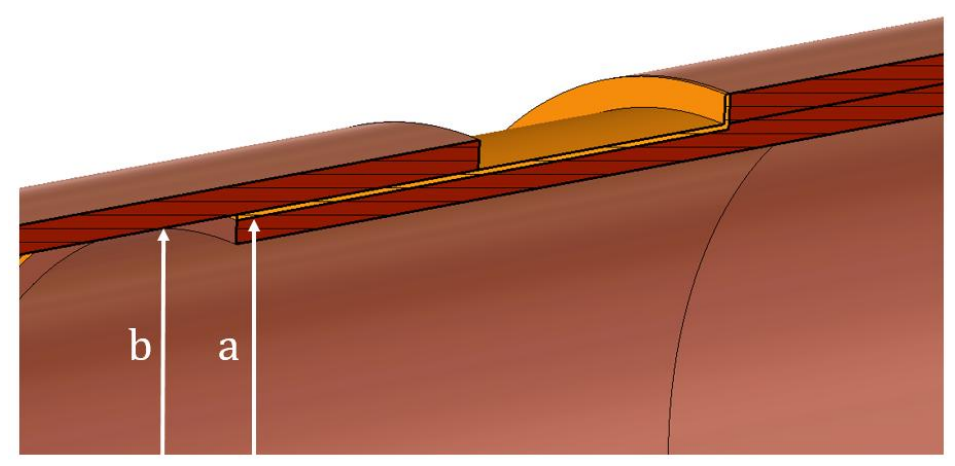

Figure 3: Cylindrical capacitor with a dielectric film.

The DC bias Tee should provide minimum reflections from the "RF" port and high transmission of the RF power towards the "RF+DC" port (see Fig. 2). The S-parameters of the capacitor can be found from $\mathrm{ABCD}$ and $\mathrm{S}$ matrices [6]:

$$
S_{11}=\frac{\frac{1}{j \omega C}}{2 Z_{0}+\frac{1}{j \omega C}}, S_{21}=\frac{2 Z_{0}}{2 Z_{0}+\frac{1}{j \omega C}},
$$

where $Z_{0}$ is the transmission line impedance, $\omega=2 \pi f$ is the angular frequency. The impedance of the QWT depends from the exact length of the Tee. Figure 4 presents minimum reflection frequency of the bias Tee as a function of the QWT length calculated by analytical formula in the presence of capacitor in the inner conductor. The length of the QWT is measured from the axis of the main coaxial line. The final adjustment of the QWT was done experimentally by moving the shorting plate and measuring $\mathrm{S}_{11}$ by the network analyzer.

The DC bias Tee was also simulated using CST Microwave Studio [7] in the frequency domain. Figure 5 summarizes results of analytical calculation, simulation and measurements (see Fig. 6) of the $S_{11}$ parameter of the both DC bias Tees. The small difference between the values of the $S_{11}$ at $322 \mathrm{MHz}$ is caused by uncertainty of the capacitance value (fringe field effects, uncertainty of Kapton film thickness, fabrication accuracy, etc.) and an assumption of loss-free QWT. The $\mathrm{S}_{11}$ on the second DC bias Tee at $322 \mathrm{MHz}$ was measured to be -41 . $\mathrm{dB}$. The lower reflection is the result of the increased capacitance. We have also measured power transmission from the RF input to the DC feedthrough installed on the QWT shorting plate. The transmission was $-50 \mathrm{~dB}$ and $-36 \mathrm{~dB}$ correspondingly for the first and the second DC bias Tee. Very low power transmission in the first DC bias Tee was achieved by installation of ferrite chokes on the DC cable as shown in Fig. 2. On the second DC bias Tee the ferrite chokes were not efficient. 


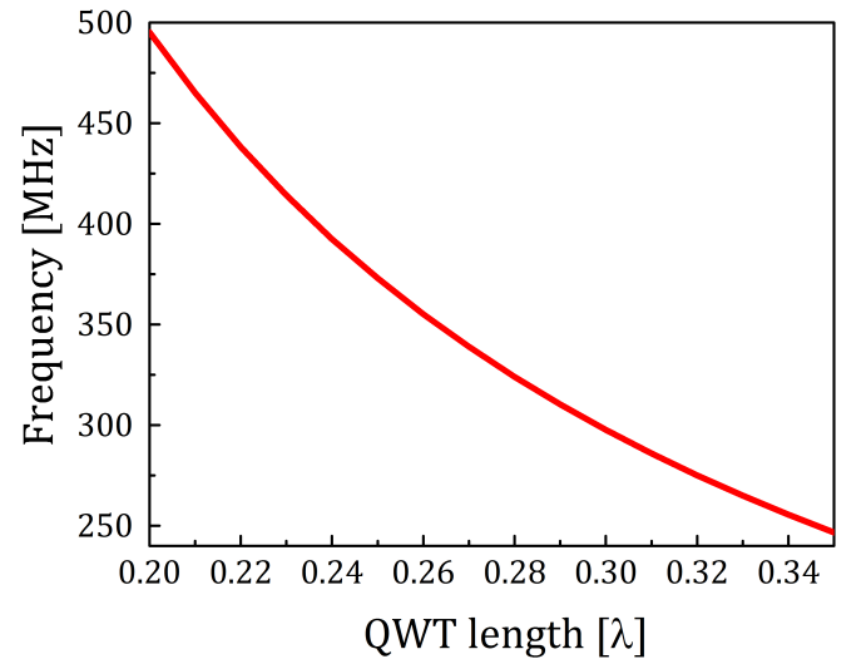

Figure 4: Tuning curve of the QWT.

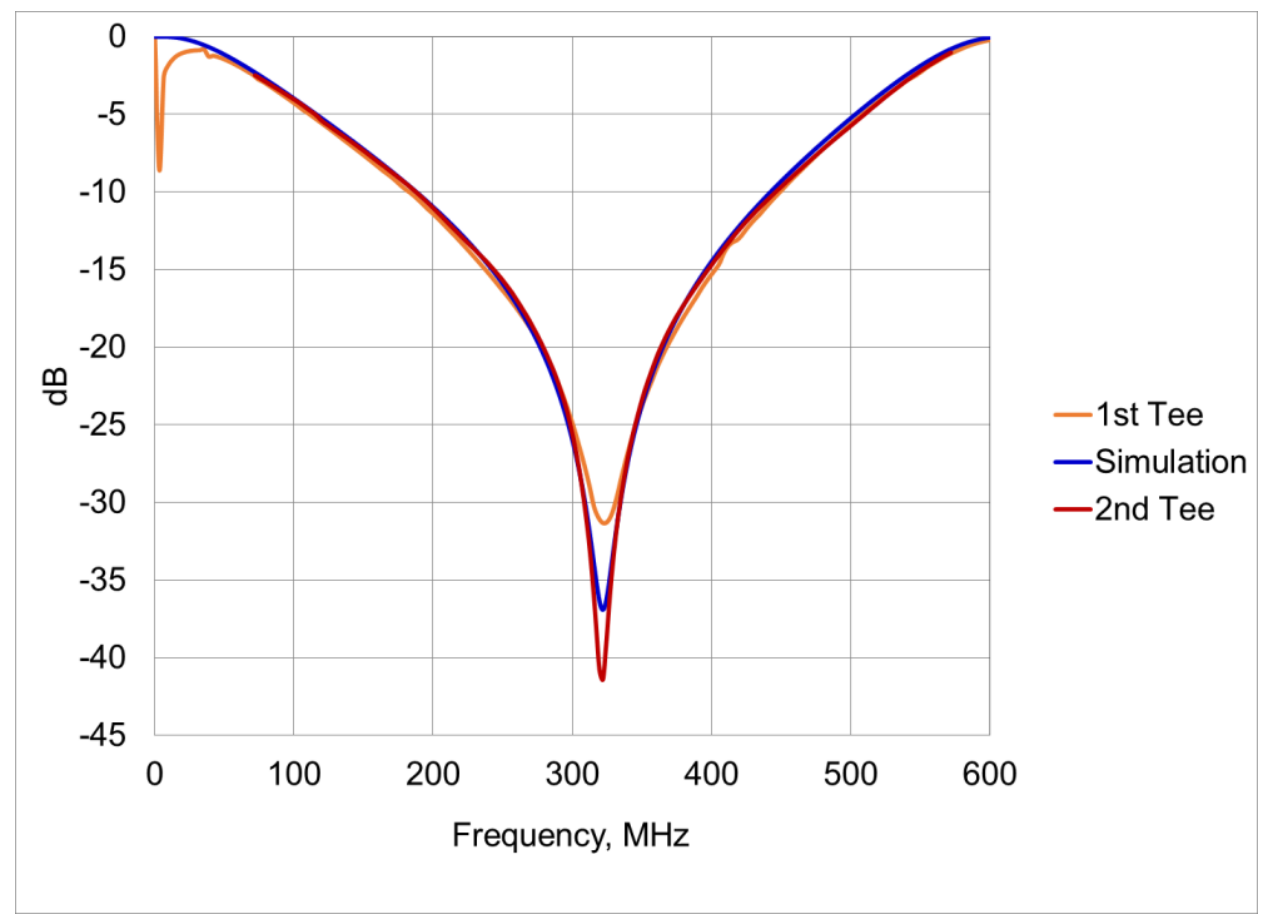

Figure 5: $\mathrm{S}_{11}$ parameter of the DC bias Tees. 


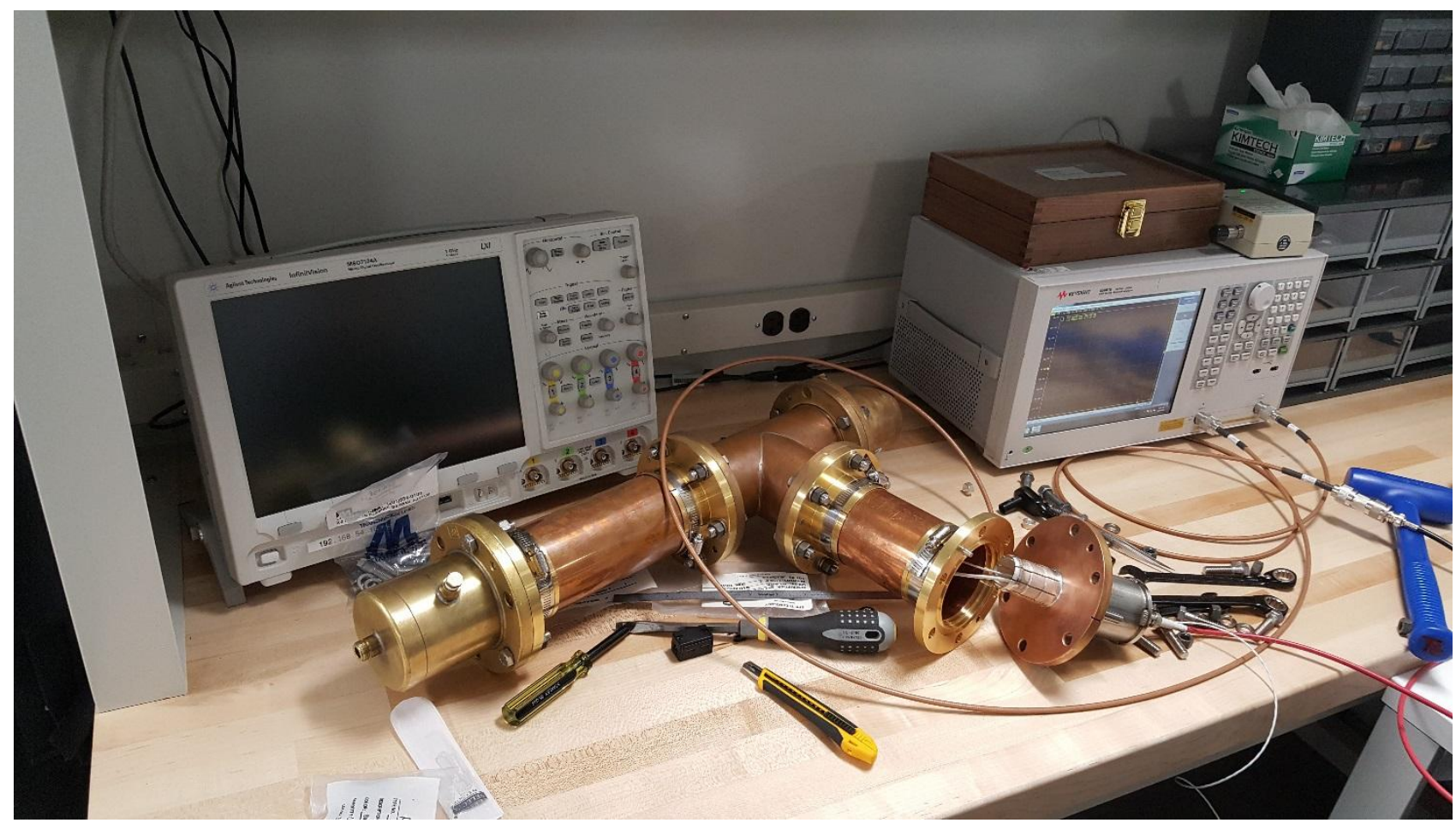

Figure 6. The DC bias Tee during the RF measurements.

\section{Off-line conditioning of SC cavities in cryomodule with DC bias Tee}

Two bias Tees have been built and installed on the RF transmission lines to permit the conditioning of two resonators simultaneously as shown in Fig. 7. As was mentioned, without the bias voltage, the RF couplers require conditioning in the full range of forward RF power. The conditioning of the couplers at cavity resonance does not guarantee the absence of multipacting when the cavity is excited off resonance. Therefore, the cavity can be phase locked and stable operation can be achieved only with the biased antenna.

A significant increase of the residual gas pressure in the coupler and temperature rise on the outer conductor are indicators of multipacting. As we found, application of $-1.0 \mathrm{kV}$ or $+1.0 \mathrm{kV}$ fully suppresses multipacting and both vacuum pressure and coupler temperature are stable. RF conditioning is required for some HWRs at accelerating gradients $E_{A C C} \geq 3 \mathrm{MV} / \mathrm{m}$ to reduce field emission. Operation with the biased RF antenna allows us to proceed to the cavity conditioning without spending any time on RF coupler conditioning. As a result all 8 cavities in the first $\beta=0.53$ cryomodule have been fully conditioned at design level of accelerating field.

The following measurements were performed to characterize the bias Tee. One of the RF couplers that had not been conditioned for multipacting was tuned to $\mathrm{Q}_{\mathrm{EXT}}=6.4 \times 10^{6}$. Then, we operated the resonator at various levels of RF power and bias voltage. During these experiments, the cavity tripped multiple times due to the rise of the pressure in the RF coupler. Figure 8 shows the variation of the coupler pressure during these measurements. Each spike in the pressure 
above $10^{-7}$ Torr interlocks the RF amplifier. The measurement results are summarized in Table 2. The resonator was operated with $5 \mathrm{~kW}$ forward power for prolonged time. The temperature sensor installed in the inner conductor near the bias Tee capacitor registered the highest temperature of $55 \mathrm{C}$ which is significantly below specification of $80 \mathrm{C}$. Based on these measurements, we have selected $-1.0 \mathrm{kV}$ as an operational value for the bias Tee for FRIB 322 MHz cavities. The new cryomodules with $322 \mathrm{MHz}$ cavities will be assembled with multipactorfree RF couplers as was discussed in ref. [1,2]. Perhaps, the bias Tees will not be required for new cryomodules.

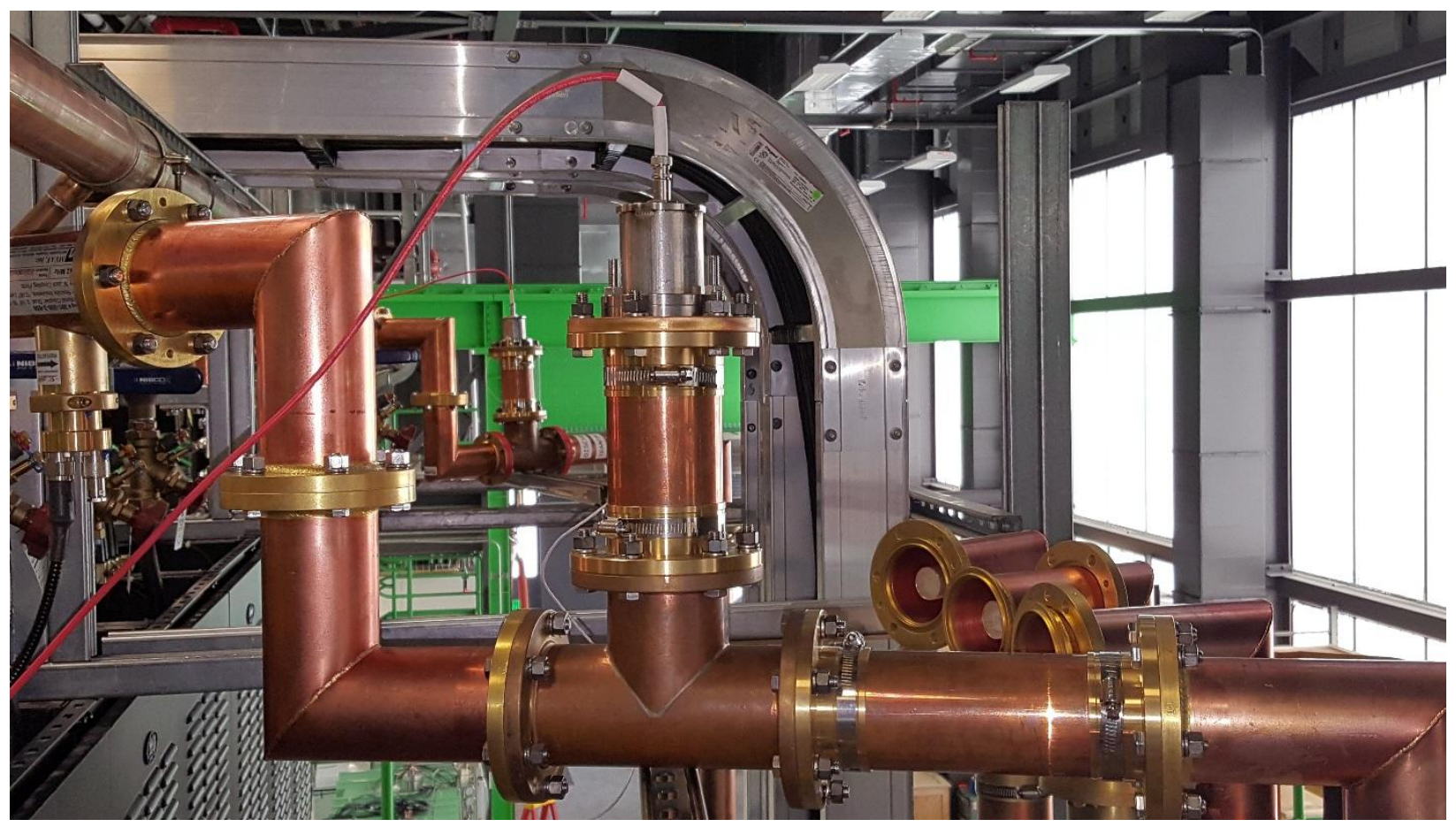

Figure 7. Two DC bias tees installed in the transmission lines from the amplifiers (on the left) to the resonators (down to right).

Table 2. Measurement results

\begin{tabular}{|c|c|c|c|}
\hline Trip case (see Fig. 8) & Bias voltage, $\mathrm{V}$ & $\mathrm{E}_{\mathrm{ACC}}, \mathrm{MV} / \mathrm{m}$ & $\mathrm{P}, \mathrm{kW}$ \\
\hline 1 & 0 & 2.85 & 0.63 \\
\hline 2 & -200 & 2.85 & 0.63 \\
\hline 3 & -300 & 4.3 & 1.25 \\
\hline 4 & -400 & 6.62 & 3.4 \\
\hline 5 & -500 & 7.03 (detuned) & 5.0 \\
\hline 6 & +200 & 4.55 & 1.6 \\
\hline 7 & +300 & 6.41 & 3.2 \\
\hline 8 & +400 & 7.95 & 5.0 \\
\hline 9 & +500 & 6.75 (detuned) & 5.0 \\
\hline
\end{tabular}




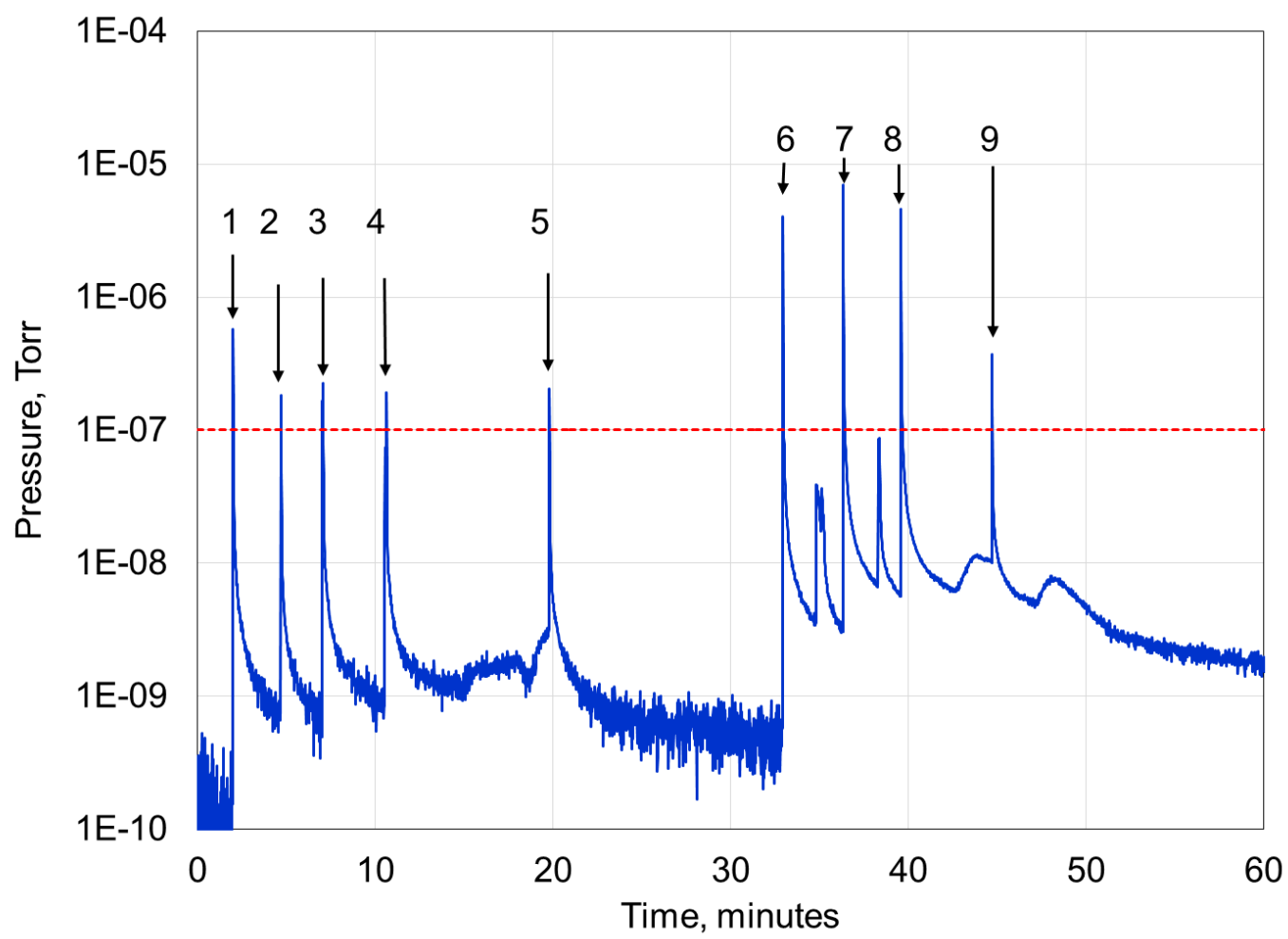

Fig.8. Pressure and temperature of the coupler as a function of time during the conditioning process. The dashed red line is the interlock limit for the RF amplifier trip due to the pressure rise. The numbers for each trip correspond to various settings of the bias voltage summarized in Table 2.

\section{Conclusion}

A device to provide a bias voltage on the RF coupler antenna has been developed, built and successfully tested. Thanks to the bias Tee the cryomodule with 8 HWRs of the FRIB SC linac was commissioned to the design specifications in a short time period. The bias Tee will be installed permanently for the first 8 resonators and, possibly, for all remaining 212 HWRs of the FRIB linac if necessary.

\section{Acknowledgements}

The authors appreciate significant help from K. Holland, S. Miller and T. Russo during the fabrication and installation of the DC bias Tees.

This material is based upon work supported by the U.S. Department of Energy Office of Science under Cooperative Agreement DE-SC0000661, the State of Michigan and Michigan State University.

\section{References}

1. Z. Zheng, A. Facco, Z. Liu, J. Popielarski, K. Saito, J. Wei, Y. Zhang, Prediction and suppression of two-point 1st order multipacting, Nuclear Instruments and Methods in Physics Research A 735 (2014) 596-601 
2. Z. Zheng, J.T. Popielarski, K. Saito, S. Stark, T. Xu, Y. Yamazaki, Design and Commissioning of FRIB Multipactor-free fundamental power coupler, Proc. of LINAC'16, September 2016.

3. S.V. Kutsaev, M.P. Kelly and P.N. Ostroumov, Design of RF power coupler for superconducting cavities, 2012 JINST 7 P11004.

4. B. Kaizer, Y. Ben Aliz, I. Fishman, J. Rodnizki, L. Weissman, Warming Rate Reduction of the SARAF RF Couplers by Application of a High Voltage De Bias, Proc. of LINAC'14, http://accelconf.web.cern.ch/AccelConf/LINAC2014/papers/thpp132.pdf, p. 1168.

5. S. Kazakov, B.M. Hanna, O.V. Pronitchev, Testing of $325 \mathrm{MHz}$ Couplers at Test Stand in Resonance Mode, Proc. of SRF'15, http://accelconf.web.cern.ch/AccelConf/SRF2015/papers/thpb098.pdf, p. 1376.

6. D. Pozar, Microwave Engineering, $4^{\text {th }}$ edition, J. Wiley \&Sons Inc.

7. https://www.cst.com/Products/CSTMWS 